\section{Determinación de la reserva ovárica mediante el recuento de folículos antrales en mujeres en edad reproductiva}

\author{
JOAQUÍN ERRÁZURIZ V. ${ }^{1,2,3}$, ÁLVARO CARRASCO', \\ ELISA DÍAZ R. ${ }^{1}$, PABLO SANHUEZA R. ${ }^{2}$, \\ PATRICIO GONZÁLEZ S. ${ }^{2}$, PATRICIO DONOSO P. ${ }^{2}$
}

\section{Association between antral follicle count and age in fertile women}

\begin{abstract}
Background: Age plays a crucial role in women fertility and is related with a decreased oocyte quality and quantity. The evidence for this asseveration comes from data obtained in infertile women. However there is a paucity of studies in patients with proven fertility evaluating ovarian reserve and its decline with time. Aim: To measure antral follicle count (AFC) in probed fertile patients and to create a distribution chart based on variables that may affect its natural decline. Material and Methods: Observational prospective study. A transvaginal ultrasound was made between menstrual cycle days 3 and 5, evaluating AFC in 100 patients, followed during a year. Results: Median antral follicle count media was 14.5 (interval 3 to 26). There was a lineal decline of AFC with age. Women with hypertension and type 2 diabetes mellitus had significantly less follicles. However, a multiple regression analysis showed that only women's age was a significant predictor of AFC $\left(R^{2}=0.84\right)$. Conclusions: There was a lineal relationship between $A F C$ and age in women with probed fertility.
\end{abstract}

(Rev Med Chile 2017; 145: 741-746)

Key words: Fertility; Ovarian follicle; Reproductive Medicine.
'Departamento de Ginecología,

Unidad de Gestión Clínica de

la Mujer y el Recién Nacido,

Hospital Padre Hurtado, Facultad

de Medicina Clínica Alemana-

Universidad del Desarrollo.

Santiago, Chile.

${ }^{2}$ Unidad de Medicina

Reproductiva Clínica Alemana.

Santiago, Chile.

${ }^{3}$ Consultorio San Rafael, La

Pintana. Santiago, Chile.

Fuentes de apoyo financiero: Se utilizó ecógrafo del consultorio.

No se contó con apoyo

financiero de instituciones $\mathrm{u}$ otras entidades.

Recibido el 3 de enero de 2017, aceptado el 27 de junio de 2017.

Correspondencia a:

Elisa Díaz

Avenida Vitacura 5951, Vitacura.

$4^{\circ}$ piso, Unidad de Medicina

Reproductiva. Clínica Alemana.

Santiago, Chile.

elisa.diaz.reiher@gmail.com
E 1 funcionamiento del ovario está determinado por la reserva ovárica, representada por el número de folículos primordiales con los que nace una mujer, y que disminuyen de manera fisiológica a lo largo de la vida reproductiva hasta la menopausia ${ }^{1,2}$. El envejecimiento del ovario parece jugar un papel trascendental en la fertilidad y se relaciona con una disminución en la calidad y cantidad de los ovocitos, que podría afectar el potencial reproductivo ${ }^{3,4}$.

Actualmente, la evaluación de la reserva ovári$\mathrm{ca}$ es de gran valor para las mujeres que reciben tratamiento por infertilidad ${ }^{5,6}$. Para lograr esta evaluación, se han descrito múltiples marcadores que pueden ser de utilidad. De estos, el recuento de folículos antrales por ultrasonido, en condiciones basales y sin estimulación ovárica, es uno de los principales exámenes. La evaluación del tamaño de la cohorte de folículos antrales con el uso de ultrasonido es factible, precisa, reproducible y puede ser una forma de estimar el estado reproductivo de una mujer ${ }^{7,8}$. Hasta la fecha, no existen estudios que demuestren una relación entre el recuento de folículos antrales con la edad cronológica en la población chilena de mujeres con fertilidad probada, lo cual tiene implicancias importantes en la planificación del embarazo y consejería en nuestro medio. El objetivo de este estudio es determinar el recuento de folículos antrales en mujeres con fertilidad probada en edad reproductiva. Secun- 
dariamente, en aquellas con deseo de embarazo, se evaluó la relación entre el recuento de folículos antrales y la ocurrencia de embarazos en un año de seguimiento.

\section{Materiales y Métodos}

El estudio se realizó en Santiago de Chile, en el consultorio San Rafael, La Pintana, entre diciembre de 2013 y diciembre de 2014. Fueron evaluadas 118 pacientes, incluyendo finalmente 100 pacientes que cumplieron con los criterios de inclusión y exclusión. Las mujeres fueron reclutadas prospectivamente, cuando asistieron para atenderse por los siguientes motivos de consulta: control por programa nacional de detección de cáncer de cuello uterino $(n=22)$, educación antenatal $(n=$ 43) y asesoramiento del dispositivo intrauterino de cobre $(\mathrm{n}=35)$. El tamaño muestral se calculó en base a la población de mujeres inscritas en el consultorio en los últimos 5 años y con controles vigentes.

Las mujeres ingresadas al estudio cumplieron con los siguientes criterios de inclusión: multíparas, ciclo menstrual regular (entre 25 y 35 días) durante los últimos 6 meses, edad entre 18 y 45 años y la presencia de ambos ovarios. Los criterios de exclusión consideraron: el uso de anticonceptivos en los últimos 6 meses, antecedentes de enfermedad inflamatoria pélvica o cirugía de ovarios, historia de infertilidad, síndrome de ovario poliquístico (diagnosticado según los criterios de Rotterdam) y enfermedades endocrinas (incluyendo hiperandrogenismo por laboratorio o examen físico en pacientes no diabéticas y pacientes con pobre visualización ecográfica de los ovarios). Se solicitó un consentimiento informado a todas las mujeres.

Después del reclutamiento, las pacientes asistieron entre el día 3 y 5 del ciclo menstrual. Se realizó una ecografía transvaginal usando un transductor de 6,5-MHz en un ecógrafo Mindray DC-6. Todas las ecografías fueron realizadas por un solo operador. Los ovarios se estudiaron con ecografía, visualizando desde el margen externo hasta el margen interno. Todos los folículos entre 2 y $10 \mathrm{~mm}$ de tamaño fueron contabilizados. El tamaño folicular fue medido utilizando los diámetros internos. El promedio de dos mediciones perpendiculares supone el tamaño folicular. La suma del recuento de ambos ovarios corresponde al recuento de folículos antrales. A cada participante se le indicó que, en caso de embarazarse, acudieran a control a la brevedad y se realizó seguimiento telefónico cada 6 meses, para consultar por embarazo. Este estudio fue aprobado por el Comité de Ética del consultorio y se realizó un consentimiento informado.

Toda la información recabada fue descargada a una base de datos en plataforma Microsoft Excel 2000 para su posterior análisis usando IBM SPSS v.22.

Los parámetros descriptivos de la población estudiada se expresaron como media y desviación estándar para variables paramétricas y como mediana y rango (mínimo - máximo), para las no paramétricas. Las frecuencias obtenidas se presentan como valores absolutos y porcentajes.

El estudio de normalidad de cada variable se llevó a cabo utilizando el test de Kolmogorov-Smirnov.

Se realizó un análisis de asociación entre recuento de folículos antrales e índice de masa corporal (IMC) (según clasificación nutricional de la Organización Mundial de la Salud [OMS] 2015, en desnutrición, normopeso, sobrepeso y obesidad tipo I, II y III); recuento de folículos antrales y presencia de tabaquismo ( 5 o más cigarros día, según OMS 2013); recuento de folículos antrales y antecedentes mórbidos (hipertensión arterial [HTA], intolerancia a hidratos de carbono [IHC], diabetes mellitus tipo 2 [DM2) [OMS 2010]). Las comparaciones entre grupos se realizaron por medio del test de Kruskal-Wallis. El análisis post hoc de comparaciones por pares para esta prueba fue realizado usando el procedimiento de Dunn con corrección de Bonferroni para comparaciones múltiples.

El recuento de folículos antrales fue contrastado con la edad cronológica de acuerdo a grupos etáreos y para la evaluación de la significancia estadística se utilizó el test de ANOVA.

Posteriormente, se estableció la correlación de estas variables y se realizó un modelo de predicción con regresión lineal y no lineal, buscando la función matemática que mejor se ajustara a la distribución muestral, según evaluación de $\mathrm{R}^{2}$ máximo y suma de errores cuadráticos. Para optimizar el modelo predictivo en relación a la posibilidad de variables confundentes analizadas previamente, se realizó una regresión múltiple a partir de la edad, 
IMC, presencia de HTA o DM2 y tabaquismo. Las asunciones de linealidad, independencia de errores, homocedasticidad y normalidad de los residuales se cumplieron para el conjunto de datos.

Para calcular los percentiles de recuento de folículos antrales de la población en relación a la edad (percentil 5, percentil 25, percentil $75 \mathrm{y}$ percentil 95) se fabricó un nomograma asociado al método de CG-LMS, usando el software LMS chartmaker v2.54 (Medical Research Council, UK).

Para todas las hipótesis se consideró un valor $\mathrm{p}<0,05$ para juzgar significancia estadística. Los datos fueron analizados con el software estadístico SPSS (v 22.0), bajo plataforma Microsoft Windows 7.

\section{Resultados}

Un total de 118 pacientes fueron reclutadas para el estudio. Después del examen de ultrasonido, 18 pacientes fueron excluidas debido a la mala visualización de los ovarios o la presencia de quistes ováricos. Los datos se obtuvieron a partir de 100 pacientes para el análisis estadístico.

La edad tuvo una media de 31 años (18-45). El recuento de folículos antrales total tuvo una mediana de 14,5 (rango entre 3 y 26 folículos), con una paridad promedio de 2 (1-5). El 12\% de la población se encontraba en normopeso, $2 \%$ con desnutrición, $4 \%$ sobrepeso y $82 \%$ con algún grado de obesidad. El 38\% de la población no presentaba patologías y en $62 \%$ se describió alguna comorbilidad (HTA, DM2, IHC, obesidad). Además, se registró $51 \%$ de tabaquismo. El resumen de las características demográficas se muestra en la Tabla 1.

Para el análisis de los resultados, las mujeres fueron categorizadas en 4 grupos etáreos:

- Grupo 1: $\leq 30$ años $(\mathrm{n}=48)$

- Grupo 2: 31-35 años $(\mathrm{n}=19)$

- Grupo 3: 36-40 años $(\mathrm{n}=20)$

- Grupo 4: 41-45 años $(\mathrm{n}=13)$

Se aplicó el test de ANOVA y se evidenció que el recuento de folículos antrales (RFA) tuvo una diferencia estadísticamente significativa entre los diferentes grupos etáreos $(\mathrm{p}<0,001)$.

$\mathrm{Al}$ evaluar la relación de patologías médicas como HTA, IHC y DM2, se evidenciaron dife- rencias estadísticamente significativas para HTA y DM2 en relación a el RFA $(\mathrm{p}<0,001)$ y no se encontró diferencias estadísticamente significativas en relación a IHC y RFA $(\mathrm{p}<0,87)$.

Se realizó el análisis de todas las variables confundentes (HTA, DM2, IHC, IMC, tabaco y edad) bajo un modelo de predicción con regresión múltiple, sin embargo, a pesar de las asociaciones evidenciadas anteriormente, solo se demostró que la edad se correlacionaba con el recuento de folículos antrales, presentando significancia estadística $\left(\mathrm{R}^{2}=0,84\right)$. Al correlacionar el RFA y la edad en los modelos de regresión, se evidenció que el modelo lineal fue el que mejor se ajustó a la distribución $(\mathrm{y}=35,67-0,59 \mathrm{x}$ edad, $\mathrm{F}(4,95)=131,72$; $\mathrm{p}<0,001 . \mathrm{R}^{2}$ ajustado $\left.=0,841\right)$ (Figura 1$)$.

Respecto al RFA e IMC, también se encontraron diferencias significativas entre los grupos (desnutrición, normopeso, sobrepeso y obesidad tipo I, II y III; $\mathrm{p}<0,001)$. El subanálisis de este grupo objetiva que las pacientes con IMC normal tienen un RFA más alto que las obesas tipo I $(\mathrm{p}<0,001)$ y tipo II $(\mathrm{p}<0,001)$.

En relación al RFA y tabaquismo, no se demostraron diferencias estadísticamente significativas $(\mathrm{p}=0,37)$.

\section{Tabla 1. Características de la población}

\begin{tabular}{|c|c|c|}
\hline \multicolumn{3}{|c|}{$\begin{array}{l}\text { Características de la población de estudio } \\
\text { (Números absolutos) }\end{array}$} \\
\hline Edad & $31(18-45)$ & \\
\hline Paridad & $2(1-5)$ & \\
\hline RFA total & $14,5(3-26)$ & \\
\hline IMC & $\begin{array}{l}\text { Desnutrición } \\
\text { Normopeso } \\
\text { Sobrepeso } \\
\text { Obesidad Tipo I } \\
\text { Obesidad Tipo II } \\
\text { Obesidad Tipo III }\end{array}$ & $\begin{array}{r}2 \% \\
12 \% \\
4 \% \\
28 \% \\
14 \% \\
1 \%\end{array}$ \\
\hline Antecedentes mórbidos & $\begin{array}{l}\text { Sana } \\
\text { HTA } \\
\text { IHC } \\
\text { DM2 } \\
\text { Obesidad }\end{array}$ & $\begin{array}{r}38 \% \\
9 \% \\
2 \% \\
8 \% \\
43 \%\end{array}$ \\
\hline Tabaquismo & $\begin{array}{l}\text { Sí } \\
\text { No }\end{array}$ & $\begin{array}{l}41 \% \\
59 \%\end{array}$ \\
\hline
\end{tabular}

En la Tabla 1 se describe epidemiológicamente a la muestra estudiada presentando edad, paridad y RFA totales en media, y describiendo los principales antecedentes mórbidos de la población chilena en porcentajes. 


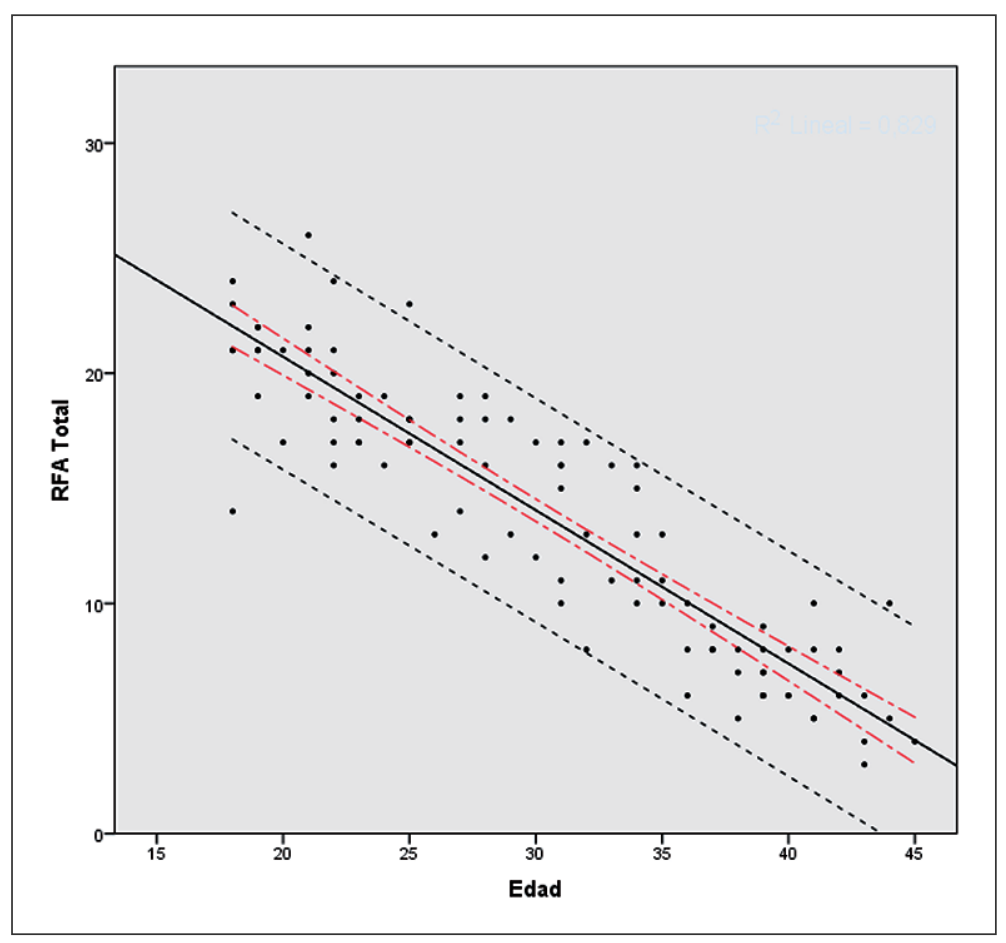

Figura 1. Correlación entre recuento de folículos antrales y edad. La línea sólida estima la mediana de RFA en función de la edad $\left(R^{2}=0,84\right)$. La línea discontinua indica los intervalos de confianza 5-95\%. La línea intercalada indica los intervalos de predicción 5-95\%. $n=100$ ).

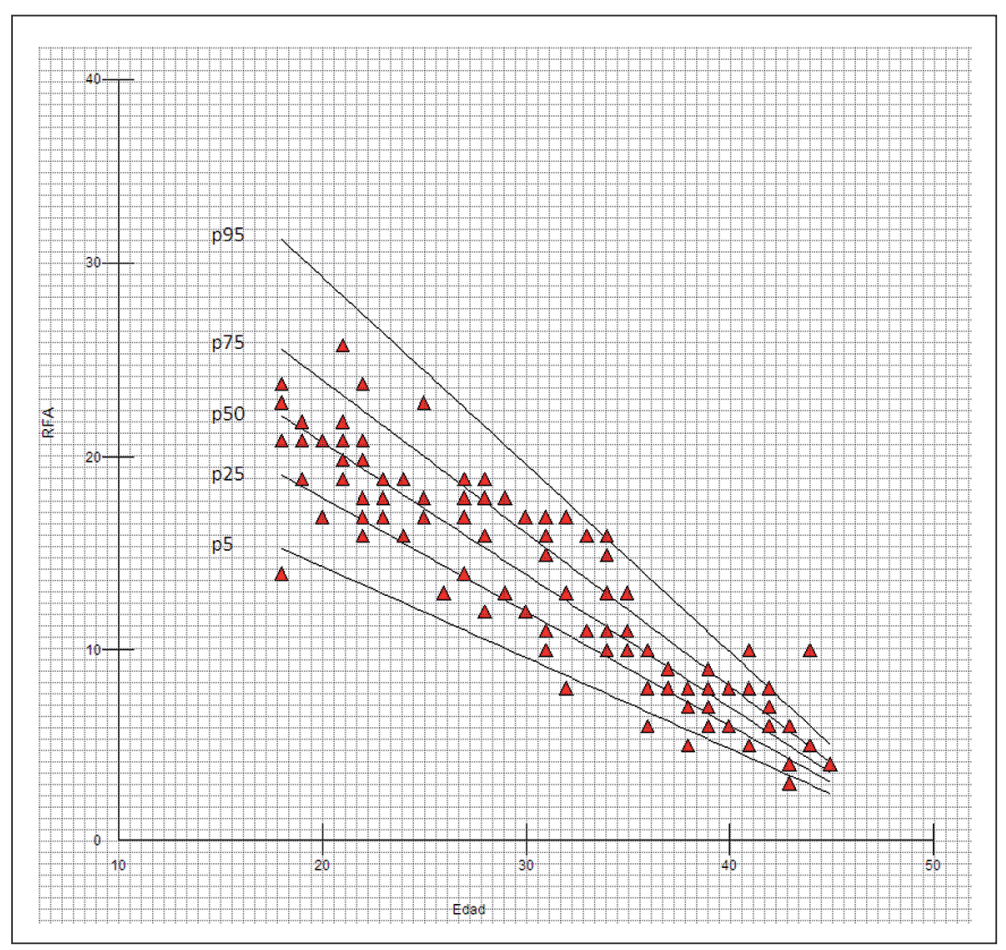

Figura 2. Percentiles de recuentos de folículos antrales específicos por edad $(5,25,50,75$ y 95). Se grafican los percentiles p5, p25, p50, p75, p95. Los triángulos representan los casos. **Por ejemplo una paciente de 20 años con 20 folículos antrales está en el percentil 25-50. 
La Figura 2 muestra el nomograma del RFA en relación a la edad, graficando las curvas de percentiles 5, 25, 50, 75 y 95 basados en el modelo CGLMS, considerando su relación lineal obtenida.

\section{Discusión}

La gran mayoría de los estudios se han enfocado en investigar el recuento de folículos antrales en pacientes infértiles ${ }^{2,10,11}$. En este estudio evaluamos la reserva ovárica de mujeres en edad reproductiva y con fertilidad probada en población chilena, lo que marca una importante diferencia con otros estudios, y nuestros resultados confirman que existe un declive en el RFA relacionado con la edad, que disminuye en forma constante durante todo el período reproductivo ${ }^{12,13}$. Scheffer et al. ${ }^{14}$ realizaron curvas que demuestran el descenso del RFA en relación a la edad, reportando un patrón bifásico, con una reducción en $4,8 \%$ anual antes de los 37 años, y de 11,7\% después de esa edad. En un segundo análisis, realizado por el grupo de La Marca et al. ${ }^{15}$, utilizaron un modelo que presentó una disminución lineal del RFA hasta la edad de 43 años. A diferencia del patrón bifásico reportado por Scheffer et al., nuestro estudio mostró un modelo lineal de pendiente única hasta los 45 años, similar a lo descrito por La Marca et al.

Al evaluar la influencia de diferentes patologías médicas en forma independiente, como HTA, IHC, DM2 y obesidad, se evidenciaron asociaciones estadísticamente significativas, que podrían incidir en el RFA, sin embargo, al utilizar la regresión múltiple para evaluar variables confundentes, solamente la edad logró significancia estadística. Esto se puede explicar dado que las pacientes con estas patologías caen dentro del grupo etáreo de mayor edad, lo que influye directamente con el RFA.

Existen múltiples estudios que asocian el consumo de tabaco con la disminución del recuento de folículos antrales ${ }^{16,17}$. Nuestro estudio no demostró diferencias en el RFA de mujeres fumadoras versus no fumadoras. No obstante, nuestro estudio no consideró el número de cigarrillos por día o la frecuencia, por lo que se requerirían estudios más acabados para sacar algún tipo de conclusión al respecto.

Nuestro estudio realiza un nomograma de RFA con descripción de percentiles en base a la población por edad, lo que permite entregar una información valiosa sobre la reserva ovárica esperable.

$\mathrm{Al}$ analizar la ocurrencia de embarazos con seguimiento de un año según el RFA, no se logró demostrar la significancia estadística de esta relación, fenómeno que podría explicarse por el bajo número de pacientes incluidas en el análisis. Sin embargo, se evidenció que todos los embarazos ocurrieron con un RFA sobre el percentil 50, lo que sugiere que mujeres con RFA bajo este percentil pudiesen requerir consejería o técnicas de reproducción asistida. De todas formas, aún se necesitan estudios con un mayor número de pacientes para probar esta hipótesis.

Dentro de las debilidades del estudio, destaca la presencia de un bajo número muestral, lo que no nos permitió obtener un poder estadístico adecuado para sacar conclusiones certeras respecto a las posibilidades de embarazo en un año de seguimiento de acuerdo al RFA. Por otra parte, la población reclutada en nuestro estudio correspondió a una muestra no representativa de nuestro país, ya que fue realizado en un consultorio al cual concurren pacientes que en su mayoría pertenece a un nivel socioeconómico bajo.

Como fortaleza, el diseño del estudio fue prospectivo, obtuvimos un seguimiento adecuado, con una pérdida menor a $20 \%$ y es el primer estudio realizado en nuestro país para determinar las curvas de normalidad en recuento de folículos antrales en mujeres en edad reproductiva. Otra fortaleza está dada por el seguimiento de un solo operador en imágenes durante todo el estudio.

Actualmente, el RFA es uno de los parámetros más comúnmente vinculados a la función ovárica. Sin embargo, hasta ahora, la interpretación de la medición se basa principalmente en la experiencia individual del operador, ya que no hay curvas de normalidad. Es por esto que el establecimiento de un nomograma con valores de RFA podría ser una herramienta útil y el primer paso para futuras investigaciones, pudiendo aportar a la medicina reproductiva en Chile.

Poder estimar la reserva ovárica es útil, no sólo para asesorar sobre las posibilidades de éxito en un tratamiento de medicina reproductiva, sino también para poder aconsejar a una pareja que desea posponer el embarazo por razones no médicas y de forma temporal. Los resultados de los marcadores de reserva ovárica no deben ser 
utilizados para excluir pacientes candidatas a tratamientos de medicina reproductiva, sino que para ofrecer asesoramiento a la pareja acerca de sus posibilidades de embarazo y poder diseñar el plan de tratamiento más apropiado ${ }^{9}$.

\section{Referencias}

1. Gougeon A, Ecochard R, Thalabard JC. Age-related changes of the population of human ovarian follicles: increase in the disappearance rate of non- growing and early-growing follicles in aging women. Biol Reprod 1994; 50 (3): 653-63.

2. Faddy MJ, Gosden RG, Gougeon A, Richardson S, Nelson JF. Accelerated disappearance of ovarian follicles in mid-life: implications for forecasting menopause. Hum Reprod 1992; 7 (10): 1342-6.

3. Lass A, Brinsden P. The role of ovarian volume in reproductive medicine. Hum Reprod Update 1999; 5 (3): 256-66.

4. Scott RT, Hofmann GE. Prognostic assessment of ovarian reserve. Fertil Steril 1995: 63: 1-11.

5. Broekmans FJ, Kwee J, Hendriks DJ, Mol BW, Lambalk CB. A systematic review of tests predicting ovarian reserve and IVF outcome. Hum Reprod Update 2006; 12 (6): 685-718.

6. De Carvalho BR, Rosa e Silva AC, Rosa e Silva JC, dos Reis RM, Ferriani RA, Silva de Sá MF. Ovarian reserve evaluation: state of the art. J Assist Reprod Genet 2008; 25 (7): 311-22.

7. Meldrum DR, Chetkowski RJ, Steingold KA, Randle D. Transvaginal ultrasound scanning of ovarian follicles. Fertil Steril 1984; 42: 803-5.

8. Scheffer GJ, Broekmans FJM, Dorland M, Habbema JDF, Looman CWN, te Velde ER. Antral follicle counts by transvaginal ultrasonography are related to age in women with proven natural fertility. Fertil Steril 1999; 72: 845-51.

9. Seifer DB, Minkoff H, Merhi Z. Putting 'family' back in family planning. Hum Reprod 2015; 30: 16-9.

10. Rosen MP, Johnstone EB, Gillham SJ, Modan AE, Lipshutz AK, Reijo-Pera R, et al. Is antral follicle count a genetic trait? Menopause 2010; 17: 109-13.

11. Hendriks DJ, Kwee J, Mol BW, te Velde ER, Broekmans FJ. Ultrasonography as a tool for the prediction of outcome in IVF patients: a comparative meta-analysis of ovarian volume and antral follicle count. Fertil Steril 2007; 87: 764-75.

12. Maseelall PB, Hernández-Rey AE, Oh C, Maagdenberg T, McCulloh DH, McGovern PG. Antral follicle count is a significant predictor of livebirth in in vitro fertilization cycles. Fertil Steril 2009; 91: 1595-7.

13. Ruess ML, Kline J, Santos R, Levin B, Timor-Tritsch I. Age and the ovarian follicle pool assessed with transvaginal ultrasonography. Am J Obstet Gynecol 1996; 174: 624-7.

14. Scheffer GJ, Broekmans FJM, Looman CWN, Blankenstein M, Fauser BCJM, De Jong FH, et al. The number of antral follicles in normal women with proven fertility is the best reflection of reproductive age. Hum Reprod 2003; 18: 700-6.

15. La Marca A, Spada E, Sighinolfi G, Argento C, Tirelli A, Giulini S, et al. Age-specific nomogram for the decline in antral follicle count throughout the reproductive period. Fertil Steril 2011; 95 (2): 684-8.

16. Berthiller J, Sasco AJ. Smoking (active or passive) in relation to fertility, medically assisted procreation and pregnancy. J Gynecol Obstet Biol Reprod (Paris). 2005 Apr; 34 Spec $\mathrm{N}^{\circ}$ 1: 3S47-54.

17. Sépaniak S, Forges T, Monnier-Barbarino P. Cigarette smoking and fertility in women and men. Gynecol Obstet Fertil 2006; 34 (10): 945-9. 